\title{
Simultaneous observations of temporally and spatially resolved electron temperatures of both circular central-cell and elliptical anchor-region plasmas in GAMMA 10
}

\author{
R. Minami, ${ }^{\text {a) }}$ T. Cho, J. Kohagura, M. Hirata, T. Numakura, M. Yoshida, H. Watanabe, \\ K. Yatsu, and S. Miyoshi \\ Plasma Research Centre, University of Tsukuba, Ibaraki, Japan
}

(Presented on 22 June 2000)

\begin{abstract}
The first results of simultaneous observations of temporally and spatially resolved electron temperatures $\left(T_{e}\right)$ in both circularly shaped central-cell and elliptically shaped anchor regions are reported in the GAMMA 10 tandem mirror. A data set is provided using a novel matrix-type x-ray semiconductor detector. The detector has seven "matrix columns" for the measurement of plasma x-ray profiles along with six "matrix rows" for simultaneous analyses of six different x-ray-energy ranges by the fabrication of six different thicknesses of $\mathrm{SiO}_{2}$ semiconductor surface layers from 1 to $495 \mathrm{~nm}$ as ultrathin and unbreakable "x-ray absorption filters." Such a matrix idea enables us to analyze x-ray tomography data in the $T_{e}$ region down to a few tens of eV. Simultaneous application of the x-ray detectors in the central-cell and the anchor region gives information on not only detailed electron behavior in each region but also the mutually communicating relation between the two regions: The role of the anchor-region plasmas in magnetohydrodynamic plasma stabilization in the central-cell plasmas is confirmed by the data from these detailed simultaneous electron observations. (C) 2001 American Institute of Physics. [DOI: 10.1063/1.1322620]
\end{abstract}

\section{INTRODUCTION}

$\mathrm{X}$-ray detection systems using semiconductor detector arrays are developed as position sensitive $\mathrm{x}$-ray imaging systems for analyzing temporally and spatially varying plasma behavior. ${ }^{1-5}$ In the GAMMA 10 tandem mirror, ${ }^{4,5}$ open ended magnetic fields produced for magnetically confined plasma formation are still strong even in the outside regions where the coils are located. Plasma diagnostic systems including x-ray detectors are placed in such high-field, limited narrow-spaced areas.

Useful characteristics of semiconductor detectors for plasma diagnostics are listed as follows. These detectors satisfy the above-described requirements of (i) compactness for narrow detector space, (ii) low-level outgassing for use in high-vacuum conditions under $10^{-8}$ Torr, as well as (iii) a high degree of immunity to ambient magnetic fields of the order of teslas. These provide remarkable merit compared to the use of other detectors including photomultipliers and microchannel plates (MCPs), ${ }^{6}$ which utilize avalanche electrons for signal outputs.

From the above several advantages, we have installed semiconductor detector arrays to carry out the first simultaneous measurements of $\mathrm{x}$ rays emitted from both circularly shaped central-cell and elliptically shaped anchor-region plasmas. A minimum- $B$ field configuration in the anchor region is produced by baseball-shaped coils for maintaining magnetohydrodynamic (MHD) plasma stability. ${ }^{5}$

Recently, progress in theoretical studies of the quantum efficiency of semiconductor x-ray detectors has been made;

${ }^{a)}$ Electronic mail: minami@prc.tsukuba.ac.jp novel findings including the invalidity of conventional standard theory of the x-ray-energy response utilized over this quarter of the century, ${ }^{7}$ as well as experimental verification of our proposed theory on the energy response ${ }^{8,9}$ have been uncovered.

In spite of the above-described usefulness and the recent steady progress in the understanding of semiconductor detector physics, it is still difficult for semiconductor x-ray detectors to determine electron temperatures $T_{e}$ in the range below $100 \mathrm{eV}$ because of $\mathrm{x}$-ray absorption due to the existence of a dead layer covering the detector surface. Furthermore, the conventional "x-ray absorption method" requires many plasma shots with good reproducibility because of the necessity of shot-to-shot changes of x-ray absorption filters.

In this article, the first report of simultaneous observations of $T_{e}$ in both central-cell and anchor regions is made by a single plasma shot alone on the basis of the combination of the above-described theoretical development of x-ray analyses and our newly proposed "matrix-type" semiconductor detector (see Sec. II). These temporally and spatially resolved x-ray analyses cover the $T_{e}$ range from a few tens of $\mathrm{eV}$ up to several tens of $\mathrm{keV}$. The characteristic features and the first applications to plasma $\mathrm{x}$-ray diagnostics are detailed.

\section{GAMMA 10 TANDEM MIRROR AND NOVEL MATRIX-TYPE SEMICONDUCTOR DETECTORS}

Plasma experiments have been carried out in GAMMA 10 [see Fig. 1(a)], ${ }^{4,5}$ which is a minimum- $B$ anchored tandem mirror with outboard axisymmetric plug and barrier cells. GAMMA 10 has an axial length of $27 \mathrm{~m}$, and the total volume of the vacuum vessel is $150 \mathrm{~m}^{3}$. The central cell has a length of $6 \mathrm{~m}$ and a limiter with a diameter of $0.36 \mathrm{~m}$, and 


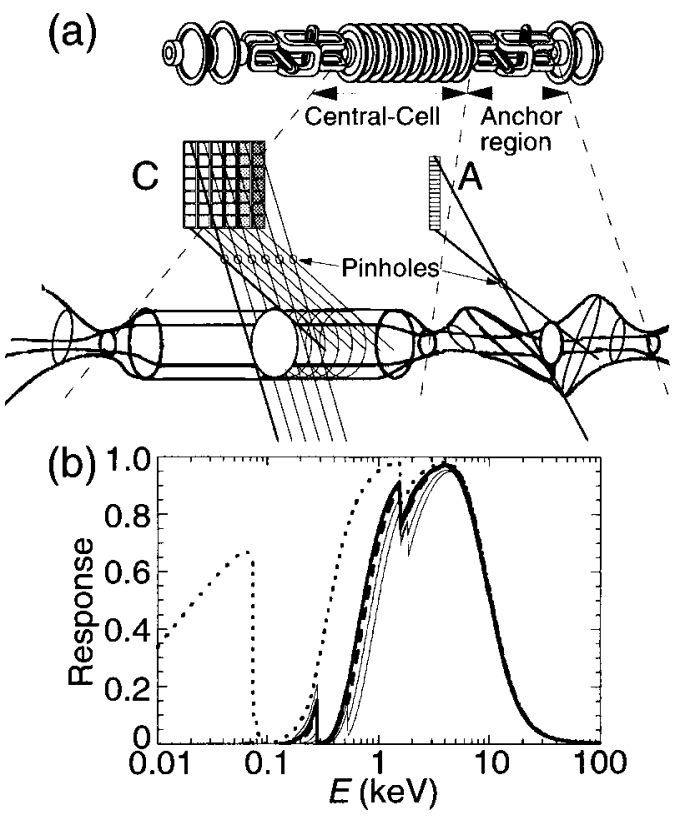

FIG. 1. Schematic drawings of (a) the magnetic coil set and magnetic-flux tube in the GAMMA 10 tandem mirror along with semiconductor $\mathrm{x}$-ray detector locations and their viewing lines of sight, labeled $\mathrm{C}$ and $\mathrm{A}$ in the central-cell and the anchor regions, respectively. (b) The x-ray-energy response of our proposed matrix-type detector in the central cell. The thick solid, the dashed, and the two thin solid curves correspond to $\mathrm{SiO}_{2}$ dead layer thicknesses of 15,110, 242, and $495 \mathrm{~nm}$, respectively, along with the absorption effect of a 1.0- $\mu$ m-thick polymer $\left(-\mathrm{C}_{8} \mathrm{H}_{8}-\right)$ filter. For reference, the dotted curve shows the fundamental response characteristics of the matrix detector in the case of a 1-nm-thick dead layer without a polymer. All these responses include the absorption property of $0.16-\mu \mathrm{m}$-thick aluminum for blocking visible light.

the magnetic field intensity at midplane is $0.405 \mathrm{~T}$ with a mirror ratio of 5.2. Ion-cyclotron heating (ICH) $(6.3 \mathrm{MHz}$, $200 \mathrm{~kW}$ ) is employed to produce hot ions in the temperature range of $1-10 \mathrm{keV}$. Two electron-cyclotron-heating $(\mathrm{ECH})$ systems in the plug region (ECH 1 and 2; $28 \mathrm{GHz}, 150 \mathrm{~kW}$ ) are prepared for producing plasma-confining potentials. Potential confined electrons are, in turn, heated due to slowing down powers from the ICH produced hot ions.

Figure 1(a) shows the magnetic coil set and magnetic flux tube in the GAMMA 10 tandem mirror. Schematic drawings of detector locations and viewing lines of sight of semiconductor $\mathrm{x}$-ray detectors are illustrated and marked by $\mathrm{C}$ and $\mathrm{A}$ in the central-cell and the anchor regions, respectively. Observations of the central-cell $\mathrm{x}$ rays are carried out by the use of a novel matrix-type semiconductor detector [see the drawings at $\mathrm{C}$ in Fig. 1(a)]. X rays are imaged on the matrix detector through six pinholes $(3 \mathrm{~mm}$ in diameter) aligned in line against the corresponding six detector rows, respectively. The spatial resolution of each detector row is 3 $\mathrm{cm}$ in the midplane of the plasmas, and the length of the totally overlapped lines of sight for the six detectors in the axial direction (parallel to $B$ ) is $6 \mathrm{~cm}$ in the midplane. This length is sufficiently short compared to the electron meanfree path; thus, the lines of sight of these six detector rows cover nearly the same region in the plasmas.

The matrix detector is fabricated on a $300-\mu \mathrm{m}$-thick $n$-type silicon wafer. The active area of each channel is 0.5 $\times 0.5 \mathrm{~cm}^{2}$, and the total area of the detector is $3.6 \times 4.1 \mathrm{~cm}^{2}$. Each detector unit is essentially a $p-n$ junction photodiode with its own output wires. It is covered with a 160 -nm-thick aluminum layer to cut out visible light. The detector is characterized by the formation of six rows with different thicknesses of thin dead layers $\left(\mathrm{SiO}_{2}\right)$ with $1,15,110,242$, and $495 \mathrm{~nm}$ on its surface compactly. Each row has seven channels (columns) for measurement of plasma $\mathrm{x}$-ray radial profiles so as to make $\mathrm{x}$-ray tomographic reconstructions.

These various $\mathrm{SiO}_{2}$ layers are proposed to be applied as "unbreakable ultrathin x-ray-absorption filters" having different thicknesses; these thin filters are never obtained as "self-sustained material absorbers" because of their ultrathin properties. This novel idea makes it possible to analyze $T_{e}$ ranging down to a few tens of $\mathrm{eV}$. The values of the depletion-layer thickness $d_{\text {dep }}$ are designed in the range of $16-21 \mu \mathrm{m}$ in our plasma experiments for the demonstration of low $T_{e}$ observations.

A tomographically reconstructed data set in various $\mathrm{x}$-ray energy ranges is simultaneously obtained from each detector row with a different ultrathin " $\mathrm{SiO}_{2}$ x-ray absorber." Consequently temporal evolution of energyresolved x-ray or $T_{e}$ profiles is conveniently attained.

The detector response of each detector row normalized by the incident x-ray energy is calculated in Fig. 1(b). Here, the effects of the detector response and the absorption effects of the 0.16- $\mu \mathrm{m}$-thick aluminum layer, as well as those of the " $\mathrm{SiO}_{2} \mathrm{x}$-ray absorber," are totally included. Thus, the energy response is defined as unity when no $x$-ray absorption in any absorption materials and no losses of $\mathrm{x}$-ray-created holeelectron pairs in a detector are assumed. The thick solid, the dashed, and the two thin solid curves correspond to $\mathrm{SiO}_{2}$ thicknesses of $15,110,242$, and $495 \mathrm{~nm}$, respectively, including the absorption effect of a 1.0- $\mu$ m-thick polymer $\left(-\mathrm{C}_{8} \mathrm{H}_{8}-\right)$ filter. For reference, the dotted curve is calculated in the case of a 1-nm-thick dead layer without a polymer filter so as to display the fundamental response characteristics of the matrix detector. Here, we may insert changeable polymer filters for high-energy x-ray analyses. One may easily fabricate higher-x-ray-energy-oriented matrix detectors by evaporating various metals onto the detector surface. Also, by the use of our novel theory of the semiconductor response, control of the impurity doping rate in the fieldfree-substrate region of a matrix detector may produce highenergy-sensitive semiconductor detectors. ${ }^{8,9}$ Such methods for controlling detector characteristics, with high efficiency from a few tens of eV up to several tens of $\mathrm{keV}$, make it possible to apply the matrix detectors to observe a wide range of $T_{e}$ including the $50 \mathrm{eV}-50 \mathrm{keV}$ region.

For checking the spatial symmetry in x-ray emission profiles and plasmas themselves, a 50 channel MCP x-ray detector, ${ }^{4,5} 16$ channel semiconductor detectors each with active channel area of $0.2 \times 0.5 \mathrm{~cm}^{2}, d_{\text {dep }}$ of $20 \mu \mathrm{m}$, and an 8 -nm-thick dead layer with a $0.5-\mu \mathrm{m}$-thick polymer filter, as well as two standard sets of $H_{\alpha}$ detector arrays and microwave interferometers are employed. These data consistently show axisymmetric plasma profiles on the basis of an $E$ $\times B$ rotation due to tandem-mirror positive potentials. Such a rigid rotation is convenient to crosscheck the azimuthal sym- 


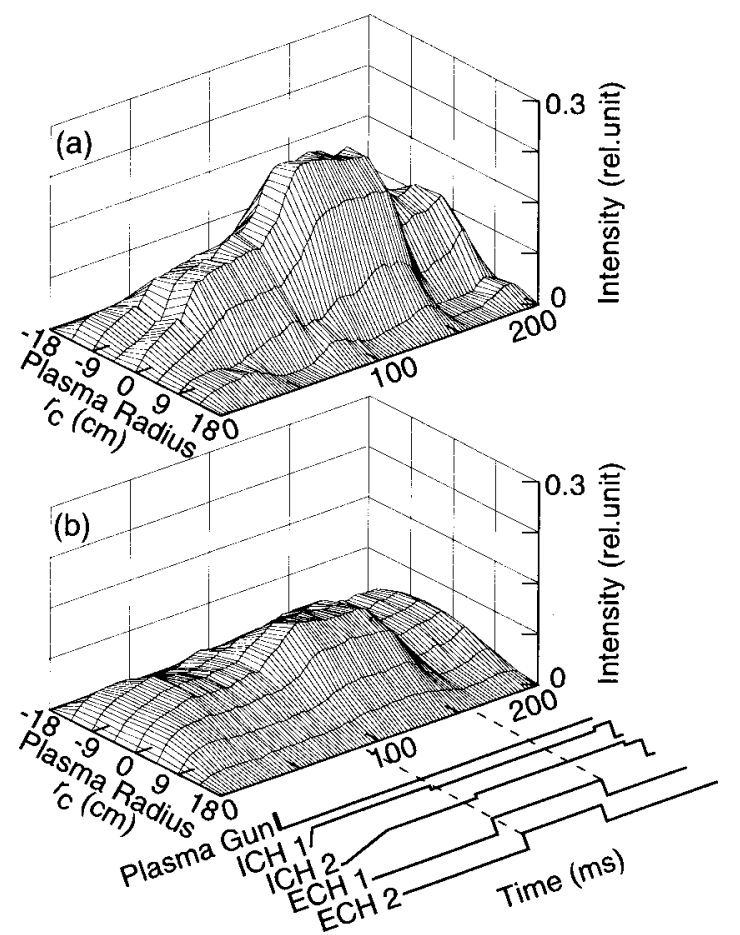

FIG. 2. Temporal evolution of the radial profiles of line-integrated $x$-ray brightness. The data in (a) and (b) from the two different detector rows, having 15- and 110-nm-thick dead layers, respectively, are simultaneously observed in the central cell of GAMMA 10. For the energy responses of data (a) and (b), one may refer to the thick solid and the dashed curves in Fig. 1(b), respectively.

metry of signals. Sixteen channel semiconductor detectors are also employed for $\mathrm{x}$-ray tomographic reconstructions in the anchor region to investigate the electron behavior and characteristics in comparison with those in the central cell.

\section{PLASMA ELECTRON-TEMPERATURE MEASUREMENTS USING THE MATRIX-TYPE SEMICONDUCTOR DETECTOR}

The temporal evolution of the radial profiles of lineintegrated x-ray intensities (brightness) in the central cell of GAMMA 10 is obtained during a single plasma shot by $\mathrm{x}$-ray signals in six different energy ranges from the six different detector rows of the matrix detector. Figure 2(a) exemplifies lower-energy $\mathrm{x}$ rays all through the plasma radii $r_{c}$, while higher-energy $\mathrm{x}$ rays in Fig. 2(b) are locally emitted from the core-plasma region. Accordingly, as a quick outlook for the two data sets, the information on a peak-onaxis $T_{e}$ profile is anticipated. For more detailed analyses, the thick solid and the dashed curves in Fig. 1(b) provide the detector responses in the cases of Figs. 2(a) and 2(b), respectively. These line-integrated data are then tomographically reconstructed for attaining spatially resolved $\mathrm{x}$-ray emissivity in plasmas. Similarly the six data sets from the matrix detector are employed for $T_{e}$ analyses.

Figure 3 summarizes the first analyzed result of temporally and spatially resolved $T_{e}$ in a single plasma shot alone using the above-described method and data (Fig. 2). As one

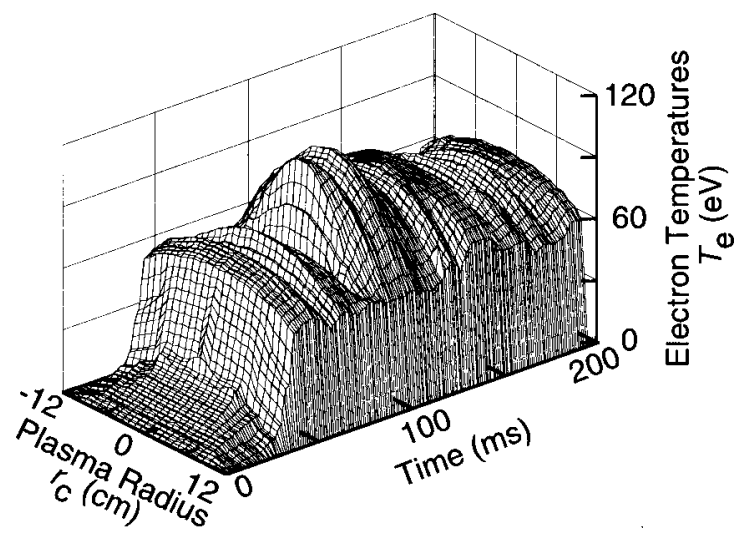

FIG. 3. Temporal evolution of spatially resolved $T_{e}$ using a single plasma shot alone. The data in Fig. 2 from the matrix detector are employed for the tomographically reconstructed data analyses.

can see by the X-ray increase during the injection period of ECH 1 and 2 in Fig. 2, the analyzed values of $T_{e}$, in fact, increase by $60 \%$ (Fig. 3) due to ECH.

\section{COMPARISON OF ELECTRON-TEMPERATURE PROFILES IN THE CENTRAL-CELL AND THE ANCHOR REGIONS DURING A SINGLE PLASMA SHOT}

As one of the applications of the above-mentioned x-ray diagnostics for investigating plasma behavior and stability, data from the matrix detector in the central cell are compared to those in the MHD stabilizing anchor region in Fig. 4 (see

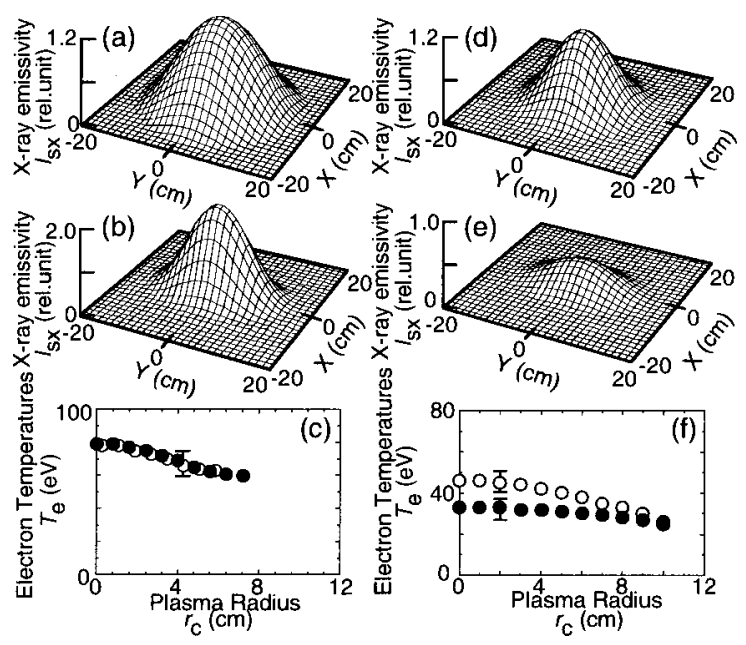

FIG. 4. Data sets (a)-(c) before and (d)-(f) after cold gas puffing in the anchor region. X-ray tomographic reconstructions in the (a), (d) central cell having a circularly shaped cross section of the magnetic-flux tube are compared to those in the (b), (e) anchor region having an elliptically shaped cross section. These data are analyzed to obtain $T_{e}$ profiles in (c) and (f) on the basis of the $\mathrm{x}$-ray absorption method by fabricating different thicknesses of unbreakable ultrathin dead layer filters in combination with polymer filters. (c), (f) Open and closed circles stand for $T_{e}$ in the central-cell and the anchor regions, respectively. The radial positions in the anchor region are mapped into those in the central cell. Various signals including microwave interferometers, $H_{\alpha}$ detectors, and end-loss ion detectors similarly show axisymmetric profiles observed with these x-ray detectors. For (a) and (d), the data in Fig. 2(a) are tomographically reconstructed. For (b) and (e), the data with an 8-nm-thick $\mathrm{SiO}_{2}$ layer and a $0.5-\mu \mathrm{m}$-thick polymer filter are, for instance, represented. 
$\mathrm{C}$ and $\mathrm{A}$, respectively, in Fig. 1). The plasma operation in Fig. 4 is modified by the use of additional gas puffing in the anchor region to cool down ICH produced hot ions due to charge-exchange ion-energy losses. The reduction of a hotion beta value in the anchor results in the decay of plasmas because of the loss of the anchor MHD stabilizer plasmas for overall GAMMA 10 plasmas. Figures 4(a)-4(c) and 4(d)4(f) represent the data sets before and after the cold gas puffing, respectively. A circularly shaped cross section of the central-cell flux tube is transformed into an elliptically shaped anchor cross section [see Fig. 1(a)]. Tomographically reconstructed $\mathrm{x}$-ray data in the central cell [Figs. 4(a) and 4(d)] and the anchor regions [Figs. 4(b) and 4(e)] are also observed to follow the shape of the cross section of the flux tube in each region [see Fig. 1(a)]. The shapes of x-ray profiles are found to be unchanged before and after the gas puffing; however, the $\mathrm{x}$-ray intensity in the anchor region is particularly reduced after gas puffing, showing the reduction of $T_{e}$ in the anchor region. Here, the axisymmetric tandemmirror positive potentials protect the core plasmas from the incidence of positively charged impurity ions, if any, along with assistance by $E \times B$ rotating motion of incident impurities in the azimuthal directions of the peripheral region. In fact, no appreciable impurity line radiation is observed in the $\mathrm{x}$-ray energy spectra using a $\mathrm{Si}(\mathrm{Li})$ detector as well as an ultralow-energy measurable pure Ge detector (10\% quantum efficiency over $200 \mathrm{eV}$ photons). Furthermore, good agreement in $T_{e}$ due to both x-ray diagnostics and electroncyclotron emission measurements confirms the abovedescribed experimental situations in GAMMA 10. (For a more detailed discussion on impurities, see Ref. 10, for example.)
X-ray analyses for $T_{e}$ profiles shown in Figs. 4(c) and 4(f) confirm the role of the anchor-region plasmas. Plasmas having $T_{e}$ beyond $50 \mathrm{eV}$ provide a good connection between the central-cell [open circles in Figs. 4(c) and 4(f)] and the anchor regions (closed circles), since the electron mean-free path $\lambda_{e}$ of $\sim 20 \mathrm{~m}$ is sufficiently longer than the distance of $5.2 \mathrm{~m}$ between both regions. After gas puffing, however, $T_{i}$ is at first cooled down, and then $T_{e}$ decreases through the reduction of slowing down powers from hot ions to electrons. The connection of the central-cell plasmas with bad curvature toward the MHD stabilizer anchor is then lost due to the lack of interchange communications between the two regions $\left(\lambda_{e} \sim 4 \mathrm{~m}\right)$. This disconnecting feature is found from difference in $T_{e}$ between the two regions shown in Fig. 4(f). Here, the radial positions in the anchor region are mapped into those in the central cell $r_{c}$ along the magnetic flux tube.

As one can see in the above examples, such plasmadiagnostics development will provide various findings of plasma-physics fundamentals, as well as applications including plasma control and stabilization.

${ }^{1}$ R. T. Snider, Rev. Sci. Instrum. 66, 546 (1995).

${ }^{2}$ B. Alper et al., Rev. Sci. Instrum. 68, 778 (1997).

${ }^{3}$ J. A. Wesson, B. Alper, A. W. Edwards, and R. D. Gill, Phys. Rev. Lett. 79, 5018 (1997).

${ }^{4}$ T. Cho et al., Phys. Rev. Lett. 64, 1373 (1990).

${ }^{5}$ T. Cho et al., Phys. Rev. A 45, 2532 (1992).

${ }^{6}$ M. Hirata et al., Nucl. Instrum. Methods Phys. Res. B 66, 479 (1992).

${ }^{7}$ W. J. Price, Nuclear Radiation Detection (McGraw-Hill, New York, 1964), Chap. 8.

${ }^{8}$ T. Cho et al., Phys. Rev. A 46, 3024 (1992).

${ }^{9}$ T. Cho et al., Nucl. Instrum. Methods Phys. Res. A 348, 475 (1994).

${ }^{10}$ T. Kondoh et al., J. Appl. Phys. 67, 1694 (1990). 